\title{
SOME CHALLENGING GROUP PRESENTATIONS
}

\author{
GEORGE HAVAS, DEREK F. HOLT, P. E. KENNE and SARAH REES
}

Dedicated to M.F. (Mike) Newman on the occasion of his 65 th birthday

(Received 15 January 1999; revised 23 April 1999)

Communicated by E. A. O'Brien

\begin{abstract}
We study some challenging presentations which arise as groups of deficiency zero. In four cases we settle finiteness: we show that two presentations are for finite groups while two are for infinite groups. Thus we answer three explicit questions in the literature and we provide the first published deficiency zero presentation for a group with derived length seven. The tools we use are coset enumeration and Knuth-Bendix rewriting, which are well-established as methods for proving finiteness or otherwise of a finitely presented group. We briefly comment on their capabilities and compare their performance.
\end{abstract}

1991 Mathematics subject classification (Amer. Math. Soc.): primary 20F05.

\section{Introduction}

The examples we consider are deficiency zero presentations; that is, presentations with the same number of generators as defining relations. Thus the presentations themselves are relatively concise, suggesting that they may be challenging for computational tools. The first example, in Section 3, is a presentation proposed by Malcolm Wicks, which is a difficult presentation of the cyclic group of order 11. The second collection of examples, in Section 4, consists of 3-generator and 3-relator presentations devised by Havas, Newman and O'Brien as candidates for deficiency zero presentations of finite $p$-groups. Most of these have been proved finite; here we show how two of them can be proved infinite; three remain unresolved. Finally, in Section 5 , we give a deficiency zero presentation for a soluble group of derived length seven.

(C) 1999 Australian Mathematical Society 0263-6115/99 \$A2.00+0.00 


\section{Computational background}

Coset enumeration is long established as a technique for the investigation of finitely presented groups. It was used well before the days of electronic computers, apparently first by Moore [22] and later systematized by Todd and Coxeter [26]. The first computer implementation was that of Haselgrove in 1953. This and other early implementations are described in [19].

In its basic usage, coset enumeration takes a finitely presented group together with a finitely generated subgroup as input, and attempts to find the index of that subgroup in the whole group. Modern variants attempt to do more, namely to find a permutation representation on the cosets of the subgroup. In principle, suitable systematic versions of coset enumeration will succeed whenever the index is finite, but in difficult examples this can take a very long time, even when the index is small. Likewise, it can use an arbitrarily large amount of space.

If the subgroup can be shown to be finite, then we have a finiteness proof for the whole group. The related Reidemeister-Schreier method can be used to construct a presentation for a finite index subgroup. Then, in suitable circumstances if a finiteindex subgroup is infinite, it is possible to prove infiniteness of the original group through the construction of infinite quotients of such a subgroup. A survey of these and other related techniques can be found in [4].

The use of the Knuth-Bendix process (originally described in [18]) as a tool for group theory was pioneered by Sims. The basic procedure (in its application to groups) is described, for example, in [6] and (in great detail) in [25]. It can be applied to any finite group presentation, and will (in principle) always succeed if the group is finite, and occasionally even when it is infinite. It is also used in combination with other methods in the study of automatic groups, as described in [5]. This can lead to a proof of finiteness or infiniteness in cases when the Knuth-Bendix process alone fails; an example of a detailed infiniteness proof appears in [14].

Coset enumeration has the advantage that it can be used most effectively to enumerate the cosets of a nontrivial subgroup of the full group. Although Knuth-Bendix can be run on the cosets of a subgroup, this often does not make it perform any better than it would on the full group. To prove finiteness, it is usually sufficient if coset enumeration completes over a cyclic subgroup of the given group. In many of the examples in Section 3, enumeration is even easier, because successful completion of coset enumeration over a 2-generator subgroup enables the theoretical deduction of the order of the group.

Experience indicates that, as a general rule, coset enumeration is much faster than Knuth-Bendix for straightforward examples. However, Sims [25, Section 5.8] points out that Knuth-Bendix is able to prove the triviality of a 3-generator 3-relator group proposed by B. H. Neumann as a challenge for computers, which no then existing 
Todd-Coxeter implementation could handle. However, if we use the six generator presentation (which makes the method of construction explicit) coset enumeration handles the group readily enough, even enumerating over the trivial subgroup.

The coset enumeration implementation we used was the one by Havas and Ramsay [10], which is an enhancement of the one described in [8]. It was run either as a stand-alone, or via an interface in the Magma system [1]. It has a wide choice of parameter settings, the most important of which indicate the relative weightings attached to defining new cosets using the coset table (traditionally called the Felsch strategy) and the relator tables (traditionally known as the HLT strategy).

The Knuth-Bendix package used was KBMAG by Holt (see [13]). This also offers a bewilderingly large choice of parameter settings. The most important is the choice of ordering for words in the generators. The default is the 'shortlex' ordering, which orders first by length and then lexicographically using the given generator order, but recursive-path orderings (a special case of 'wreath product' orderings; see [25, pp. 46-501) often produce the best performance for finite groups.

\section{The Wicks group}

Our first example is a group which appeared in the Group Pub Forum [7] in 1996. D. L. Johnson reported that coset enumeration left a 1987 question of Malcolm Wicks unanswered.

THEOREM 1. The group $W=\left\langle x, y \mid x^{3} y^{4} x^{5} y^{7}=1=x^{2} y^{3} x^{7} y^{8}\right\rangle$ is cyclic of order 11 .

We believe that Wicks' group $W$ was first proved to be finite by P. E. Kenne, who used a combination of Knuth-Bendix and Todd-Coxeter. As far as we know, this was the first use of such a composite technique to solve a previously unsolved problem. For example, one successful strategy was to use Knuth-Bendix to generate 500 new relations in $W$, and then to use coset enumeration with respect to the subgroup $\langle y\rangle$ using all of these relations. This completed successfully, with the result $|W:\langle y\rangle|=1$, after defining nearly 10 million cosets. From this it follows immediately that $G$ is cyclic, and then we can deduce $|G|=11$ by abelianising the presentation. So from the point of view of the first successful solution of an open problem, this represents a success for the combined approach.

However, further experiments by Kenne and others suggest that this particular example is solved much more easily in terms of both time and space by using KnuthBendix alone. Using a default shortlex ordering, it completes in about 360 seconds (on a SparcStation 20) after finding about 26700 relations. The recursive-path ordering used with a suitable length restriction on stored relations often yields a superior performance on finite groups, and in this case it reduced the time to 162 seconds. 
In retrospect the Wicks group can be handled by coset enumeration alone. Using principles due to Mendelsohn [21] for relator table definitions (where cosets are applied to all cyclic permutations of the relators and their inverses) we find that enumeration over $\langle y\rangle$ can be done yielding index 1, defining less than a total of 22 million cosets with suitable parameter settings. We are grateful to Colin Ramsay for pointing out the perhaps counter-intuitive result that coset enumeration over the smaller subgroup $\langle x\rangle$ (that is, the trivial subgroup) is easier: this can be done after defining a total of about 14 million cosets.

\section{Some 3-generator examples of deficiency zero}

Havas, Newman and O'Brien [9] defined fourteen groups as part of a systematic search for finite 3-generator 3-groups of deficiency zero. They are all easily shown to have a maximal nilpotent quotient of order $3^{8}$ by use of the $p$-quotient program. (In fact, by using his implementation of the Plesken soluble quotient algorithm described in [23], Brückner [2, pp. 44-48] has shown that their maximal soluble quotients all have order $3^{8}$.)

Havas, Newman and O'Brien show that the first ten groups indeed have order $3^{8}$ using coset enumeration. They report that the first five groups yield to coset enumeration over the trivial subgroup, the next four to coset enumeration over a cyclic subgroup, and the tenth to coset enumeration over a metacyclic subgroup (plus a theoretical argument). With the availability of improved computer facilities (for example, the SGI Origin 2000 computer at the University of Queensland which allows definition of hundreds of millions of cosets for these examples) the first eight can now all be enumerated over the trivial subgroup, and the next two over cyclic subgroups.

We attempted to find better proofs of finiteness for the first ten groups using Knuth-Bendix, perhaps using a combination with coset enumeration as in Section 3. We planned to go on to attack the remaining four. However, the performance of Knuth-Bendix on these examples was rather disappointing. With some difficulty, we did obtain completion with Knuth-Bendix in the first eight examples using default parameter settings. However, despite trying a wide variety of word-orders and other parameters, with both KBMAG and the Rutgers Knuth-Bendix Package, Knuth-Bendix has not yet completed with any of the remaining presentations.

In spite of its lack of success with the two hardest groups which have been proved to be finite in this collection, Knuth-Bendix has an alternative role to play. We study \#14 of [9] and prove that it presents an infinite group.

THEOREM 2. The group

$$
\left\langle a, b, c \mid a^{3}=\left[c^{-1}, b\right], b^{3}=a^{-1} c a b c^{-1} b^{-1}, c^{3}=\left[a^{-1}, b^{-1}\right]\left[a^{-1}, c\right][b, c]\right\rangle
$$


is infinite.

PROOF. By using the automatic groups program in KBMAG in the same manner as for the proof of infiniteness of $F(2,9)$ in [12], we succeed in proving that \#14 is automatic, and then deducing that it is infinite by consideration of the language of its word-acceptor. (The word-acceptor has 2889 states and each of the multiplier automata has about 8000 states.)

We have not yet made any progress (via either coset enumeration or Knuth-Bendix based techniques) towards proving finiteness or infiniteness of the groups \#11, \#12 and \#13 of [9], all of which have a maximal soluble quotient of order $3^{8}$. However, we have had further success with another example suggested in the same paper [9], which has maximal nilpotent quotient of order $5^{8}$.

THEOREM 3. The group $\left\langle a, b, c \mid a^{2} c a^{3} b^{-1} c^{-1} b, a^{-1} b a b c b^{3} c^{-1}, c b c^{2} a^{-1} b^{-1} c a c\right\rangle$ is infinite.

ProOF. Again using the automatic groups program in KBMAG, we succeed in deducing that this group is infinite by consideration of the language of its word-acceptor. (The word-acceptor has 1641 states and each of the multiplier automata has about 4000 states.)

This disposes of one candidate for a 3-generator, 3-relator group of order $5^{8}$. There are many others: the group $\left\langle a, b, c \mid a^{2} a^{3 b c}, b^{c a^{-1}} b^{4}, c^{a b} c^{4}\right\rangle$ passes all of the filters in [9], but we have not yet made any progress towards proving finiteness or infiniteness of this group. (Our inability to prove such a candidate 5-group finite is hardly surprising; no finite $p$-groups for $p>3$ are known which have 3-generator, 3-relator presentations.)

\section{A soluble group with derived length seven}

In response to a problem posed by Johnson and Robertson [15, Question 1] and [20, Problem 8.12], efficient presentations for soluble groups with increasing soluble length have been investigated by Kenne [16]. The following example provides the first published deficiency zero presentation of a group with derived length seven.

THEOREM 4. The group $G_{7}=\left\langle x, y \mid x^{4} y^{-3}, x^{-2} y^{-1} x^{-1} y^{-1}(x y)^{2} x y^{-1} x y\right\rangle$ is finite and has derived length seven.

PROOF. Coset enumeration shows that the subgroup generated by $x$ has index $2^{7} 3^{8}$ in the central quotient $G_{7}^{*}$ of $G_{7}$ obtained by replacing the first relator with the two 
relators $x^{4}$ and $y^{3}$. This suffices to show that $G_{7}$ is finite. The order of $G_{7}$ may be determined by constructing a presentation for the derived subgroup of $G_{7}$ (which has index 3) and performing a coset enumeration over the trivial subgroup of that presentation. Thus $G_{7}$ has order $2^{10} 3^{9}$. The derived length of $G_{7}$ was found by using the ANU SQ algorithm available in GAP [24].

With more computer memory it is possible to directly determine the order of $G_{7}$ : using a default strategy for hard enumerations, coset enumeration over the trivial subgroup defines the 20155392 cosets in the same maximum table size and with a total of 21168228 cosets.

The presentation for $G_{7}^{*}$ (and hence $G_{7}$ ) was found by using a variant of a method described by Campbell and Robertson [3, p. 2645], starting with a pair of permutation generators having orders 3 and 4 which generate a group having derived length six. This is not a minimal generating pair in the sense of [3].

Other presentations for finite groups having derived length seven are also known. For example, a deficiency one presentation is given in [17, p. 64], and Kenne [unpublished] has found a deficiency zero presentation for the direct product of $G_{7}$ and a cyclic group of order five.

\section{Conclusions}

We have studied some challenging group presentations and answered hard questions about four groups of deficiency zero. We have used coset enumeration and KnuthBendix rewriting as computational tools. Our examples show that both methods have important roles to play. In some cases where both methods are applicable one shows superior performance to the other in terms of use of computer resources. Furthermore there are situations where answers can be most efficiently obtained using a combination of these tools and others.

In situations where one or other of these computational methods is used to prove finiteness or infiniteness of a group for the first time, then one could say that it is that method which has been the most successful, even if other methods or parameter settings later turn out to do the job more efficiently. Both coset enumeration and Knuth-Bendix have a large number of controlling parameters, which can have huge effects on performance, and a report on such considerations for coset enumeration is in [11]. If we carry out lengthy experiments, trying different values of these parameters, on an example for which we know the answer already, then we may eventually find some particular setting that completes unusually quickly. This does not increase our mathematical knowledge, but it is an approach to comparing the relative effectiveness of the two methods which can provide the user with experience that may be useful later on new examples. 


\section{Acknowledgements}

We are grateful to the referee whose thoughtful comments led to improvements to the paper. The first author thanks the University of Newcastle, UK and the Australian Research Council for financial support during the period of this research.

\section{References}

[1] W. Bosma, J. Cannon and C. Playoust, 'The MaGMa algebra system I: The user language', J. Symbolic Comput. 24 (1997), 235-265.

[2] H. Brückner, Algorithmen für endliche auflösbare Gruppen und Anwendungen (Ph.D. Thesis, Aachener Beiträge zur Mathematik, Band 22, Verlag der Augustinus Buchhandlung, 1998).

[3] C. M. Campbell and E. F. Robertson, 'Presentations for the simple groups $G, 10^{5}<|G|<10^{6}$, Comm. Algebra 12 (1984), 2643-2663.

[4] J. Cannon and G. Havas, 'Algorithms for groups', Austral. Computer Journal 24 (1992), 51-60.

[5] D. B. A. Epstein, D. F. Holt and S. E. Rees, 'The use of Knuth-Bendix methods to solve the word problem in automatic groups', J. Symbolic Comput. 12 (1991), 397-414.

[6] R. H. Gilman, 'Presentations of groups and monoids', J. Algebra 57 (1979), 544-554.

[7] Group-pub-forum e-mail list, group-pub-forumemaths . bath .ac . uk, http://www2 .bath.ac.uk/ masgcs/problem/problem11.html, Problem 11, 1996.

[8] G. Havas, 'Coset enumeration strategies', in: Proceedings of the 1991 International Symposium on Symbolic and Algebraic Computation (ACM Press, New York, 1991) pp. 191-199.

[9] G. Havas, M. F. Newman and E. A. O'Brien, 'Groups of deficiency zero', in: Geometric and computational perspectives on infinite groups, DIMACS Series in Discrete Mathematics and Theoretical Computer Science 25 (1996) pp. 53-67.

[10] G. Havas and C. Ramsay, Coset enumeration: ACE version 3 (1999), Available as http: //www. csee. uq. edu. au/ havas/ace3.tar.gz.

[11] — 'Experiments in coset enumeration', Centre for Discrete Mathematics and Computing Report 13 (The University of Queensland, 1999).

[12] D. F. Holt, 'An alternative proof that the Fibonacci group $F(2,9)$ is infinite', Experiment. Math. 4 (1995), 97-100.

[13] — KBMAG Knuth-Bendix in monoids and automatic groups, software package (1996), Available from $\mathrm{ftp}$. warwick. maths . ac . uk in directory people/dfh/kbmag2.

[14] D. F. Holt and D. F. Hurt, 'Automatic coset systems and subgroup presentations', J. Symbolic Comput. 27 (1999), 1-19.

[15] D. L. Johnson and E. F. Robertson, 'Finite groups of deficiency zero', in: Homological group theory, London Math. Soc. Lecture Note Ser. 36 (Cambridge University Press, Cambridge, 1979) pp. 275-289.

[16] P. E. Kenne, 'Some new efficient soluble groups', Comm. Algebra 18 (1990), 2747-2753.

[17] —- Minimal group presentations: a computational approach (Ph.D. Thesis, Australian National University, 1991).

[18] D. E. Knuth and P. B. Bendix, 'Simple word problems in elementary algebra', in: Computational problems in abstract algebra (Pergamon Press, Oxford, 1970) pp. 263-297.

[19] J. Leech, 'Coset enumeration on digital computers', Proc. Camb. Phil. Soc. 59 (1963), 257-267. 
[20] V. D. Mazurov and E. I. Khukhro (eds.), The Kourovka notebook: unsolved problems in group theory, 13th Edition (Akad. Nauk SSSR Sibirsk. Otdel., Inst. Mat., Novosibirsk, 1995).

[21] N. S. Mendelsohn, 'An algorithmic solution for a word problem in group theory', Canad. J. Math. 16 (1964), 509-516 Correction, Canad. J. Math, 17 (1965) 505.

[22] E. H. Moore, 'Concerning the abstract groups of order $k$ ! and $\frac{1}{2} k$ ! holohedrically isomorphic with the symmetric and the alternating substitution-groups on $k$ letters', Proc. London Math. Soc. 28 (1897), 357-366.

[23] W. Plesken, 'Towards a soluble quotient algorithm', J. Symbolic Comput. 4 (1987), 111-122.

[24] M. Schönert et al., GAP - Groups, algorithms, and programming, Lehrstuhl D für Mathematik, 5th Edition (Rheinisch Westfälische Technische Hochschule, Aachen, Germany, 1995).

[25] C. C. Sims, Computation with finitely presented groups, Encyclopedia of Mathematics 48 (Cambridge University Press, Cambridge, 1994).

[26] J. A. Todd and H. S. M. Coxeter, 'A practical method for enumerating cosets of finite abstract groups', Proc. Edinburgh Math. Soc. 5 (1936), 26-34.

Centre for Discrete Mathematics and Computing

Computer Science and Electrical Engineering

University of Queensland, Qld 4072

Mathematics Institute

Australia

e-mail: havas@csee.uq.edu.au

University of Warwick

Coventry CV4 7AL

UK

e-mail: dfh@maths.warwick.ac.uk

Electrical and Electronic Engineering

Mathematics and Statistics

The University of Adelaide

SA 5005

University of Newcastle

Australia Newcastle upon Tyne NE1 7RU

e-mail: pek@dvcr.adelaide.edu.au

UK

e-mail: sarah.rees@newcastle.ac.uk 